\title{
Neuroglial Choristoma of the Middle Ear with Massive Tympanosclerosis: A Case Report and Literature Review
}

\author{
Hyun Joon Shim ${ }^{1}$, Yong Kyung Kang ${ }^{1}$, Yong-Hwi An ${ }^{1}$, and Young Ok Hong ${ }^{2}$ \\ ${ }^{1}$ Departments of Otorhinolaryngology-Head and Neck Surgery, ${ }^{2}$ Pathology, Eulji University School of Medicine, Eulji Medical Center, \\ Seoul, Korea
}

\author{
Received May 17, 2016 \\ Revised July 27, 2016 \\ Accepted August 16, 2016
}

Address for correspondence

Hyun Joon Shim, MD, PhD

Department of Otorhinolaryngology-

Head and Neck Surgery,

Eulji University School of Medicine,

Eulji Medical Center,

68 Hangeulbiseok-ro, Nowon-gu,

Seoul 01830, Korea

Tel +82-2-970-8276

Fax +82-2-970-8275

E-mail eardoc11@naver.com
Neuroglialchoristoma is a rare cerebral heterotopia typically involving extracranial midline structures of the head and neck, including the nose, nasopharynx and oral cavity. It rarely involves non-midline structures, such as the middle ear, mastoid and orbit. We report the case of a 63-year-old woman with right-sided hearing loss and aural fullness who was diagnosed with neuroglialchoristoma of the middle ear and mastoid. To our knowledge, this is the first report on neuroglialchoristomawith massive tympanosclerosis. The presence of combination supported the inhalation theory of neuroglialchoristoma, given that tympanosclerosis is typically caused by Eustachian tube dysfunction.

J Audiol Otol 2016;20(3):179-182

KEY WORDS: Choristoma $\cdot$ Middle ear $\cdot$ Neuroglia $\cdot$ Encephalocele.

\section{Introduction}

Choristoma is the presence of a histologically normal tissue in an abnormal location. A mature brain tissue present at a site isolated from the cranium or spinal cord is termed as neuroglialchoristoma or heterotopic neuroglial tissue [1]. Neuroglialchoristomas usually occur in extracranial midline structures, such as the nasopharynx, nose and oral cavity, and are rarely located in non-midline extracranial structures, such as the middle ear, mastoid and orbit. Although the pathogenesis of neuroglial choristomas of the middle ear is unclear, brain tissue herniation followed by absorption of their connection with the brain tissue is the most accepted hypothesis. So, the only difference between neuroglialchoristomas and encephaloceles is that the former lack a direct connection with the central nervous system [2]. We recently experienced a case of neuroglialchoristoma of the middle ear and mastoid antrum

This is an Open Access article distributed under the terms of the Creative Commons Attribution Non-Commercial License (http://creativecommons.org/licenses/by-nc/3.0/) which permits unrestricted non-commercial use, distribution, and reproduction in any medium, provided the original work is properly cited. with massive tympanosclerosis. To our knowledge, no cases on this combination have been previously reported and this combination mightsupport the inhalation theory of neuroglialchoristoma, given that poor aeration of the middle ear is a major cause of tympanosclerosis [3]. We report a case of neuroglialchoristoma of the middle ear with massive tympanosclerosis and a review of the relevant literature.

\section{Case Report}

A 63-year-old woman presented with right-sided hearing loss, which persisted since her early childhood. There was no history of congenital anomalies, trauma or ear surgery. Otoscopic examination revealed a moderate-sized tympanic membrane perforation of the right ear and small-sized tympanic membrane perforation of the left ear. Pure tone audiometry showed mixed-type hearing loss in both ears. Computed tomography revealed a soft tissue density with massive calcification in the middle ear, leading to the suspicion of bony erosions in the tegmen tympani of the right ear (Fig. 1). During canal-wall-down mastoidectomy and tympanoplasty, a well- 
cleavable vascular mass resembling a schwannoma was detected in the middle ear and mastoid antrum (Fig. 2A). A subsequent frozen section resulted in differential diagnoses, including an inflammatory lesion with fibrous stroma, a benign spindle-cell lesion, such as schwannoma, and possibly a neuroglial tissue (Fig. 3A). Because the stromal cellularity is very low and insufficient to identify a spindle-cell lesion, the presence of a schwannoma was less likely. Based on the assessment of the intraoperative frozen sections, an inflamma- tory lesion showing fibrous stroma or a neuroglial tissue was diagnosed despite its rare occurrence. The incus and stapes were fixed by massive tympanosclerosis in the middle ear (Fig. 2B). Following the incudostapedial joint separation, the incus and tympanosclerosis were excised. Although tegmen erosion over the attic was revealed during surgery, no cerebrospinal fluid (CSF) leaks or connections with the brain were observed. The tumour mass was well-separated from the intact facial canal in its tympanic segment. The final diag-
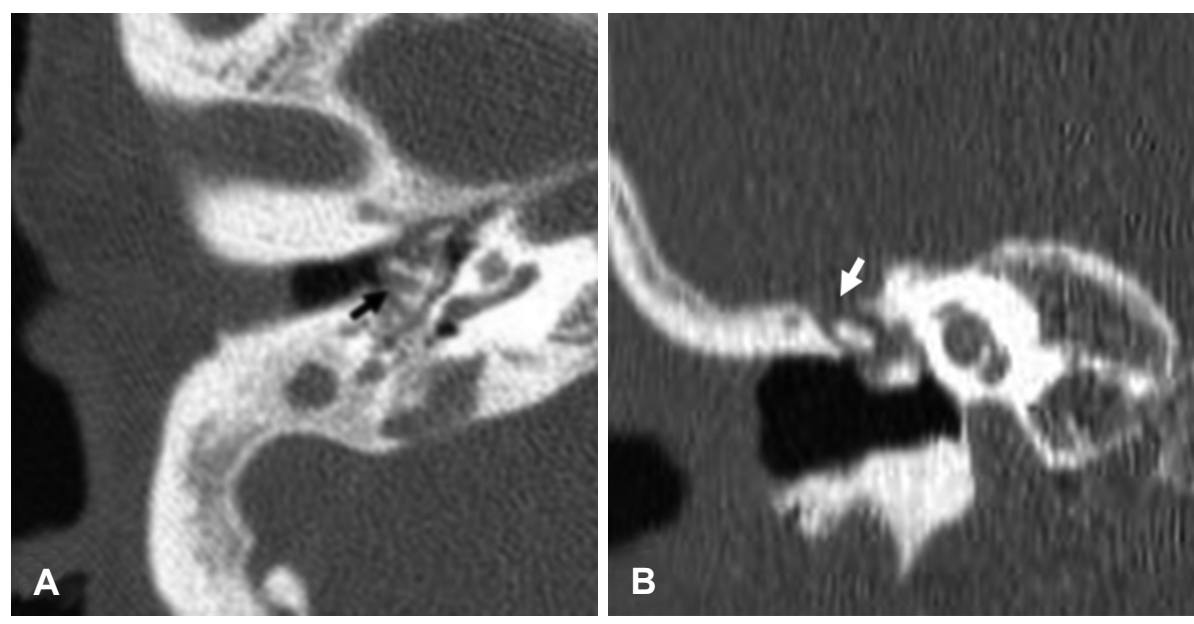

Fig. 1. A: Axial temporal bone computed tomography demonstrates soft tissue density with massive calcification in the middle ear (black arrow). B: Coronal temporal bone computed tomography demonstrates bony erosion of the tegmen tympani (white arrow).
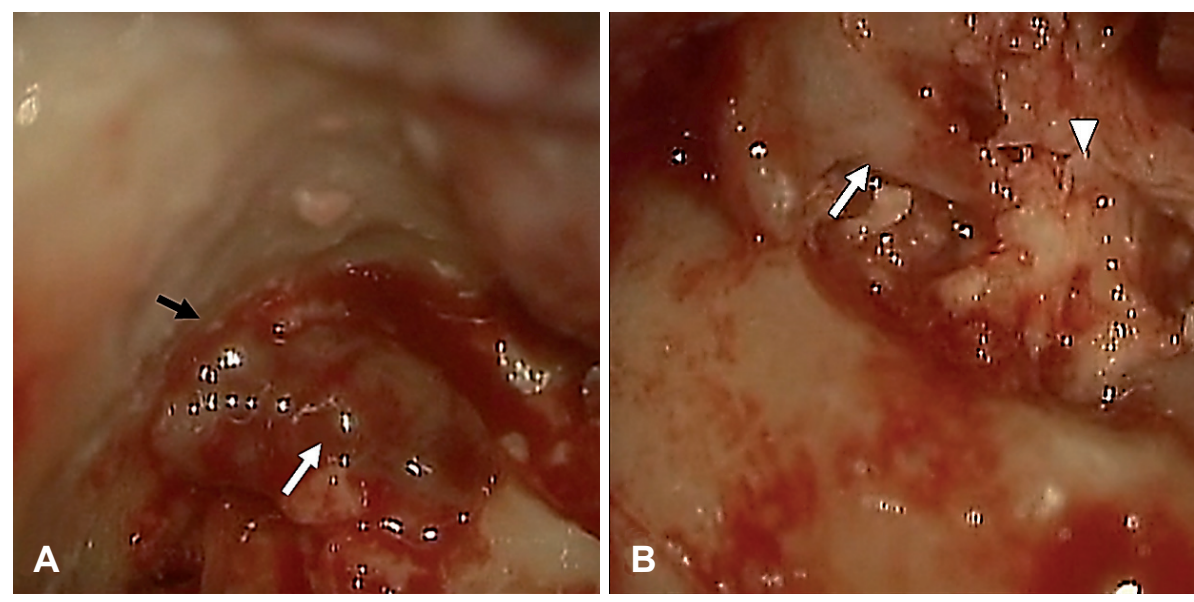

Fig. 2. A: Intraoperative findings. Tegmen (black arrow) and a wellcleavable mass (white arrow) in the epitympanum. B: Incus (white arrow) aggregating massive tympanosclerotic plaque (white arrowhead) in the mesotympanum.
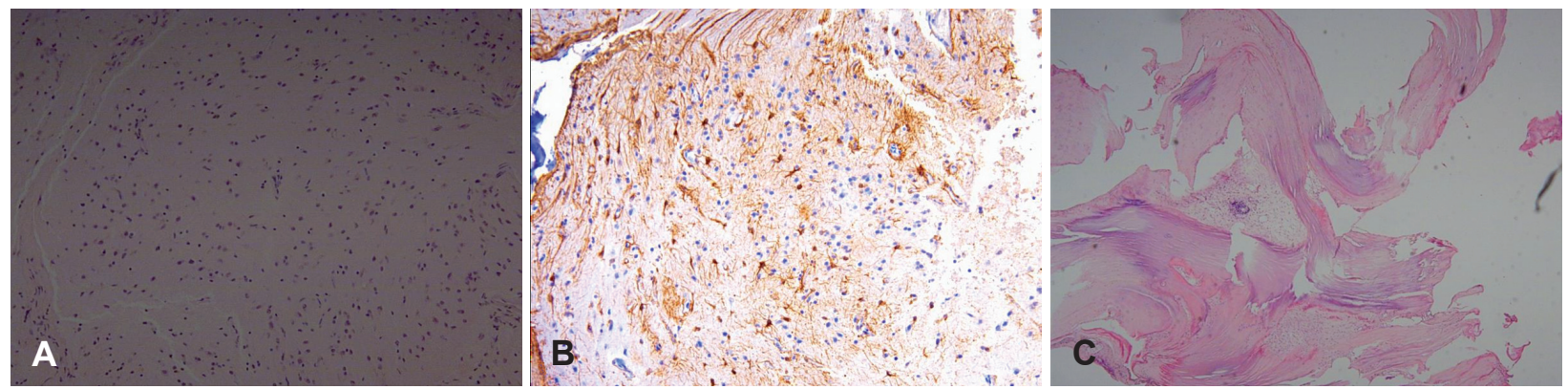

Fig. 3. Histologic features of the lesion. A: The frozen biopsy specimen comprising scattered mononuclear cells of various sizes and shapes against a fibrillary background $(H \& E, \times 200)$. B: Immunohistochemical staining revealed immunopositivity for glial fibrillary acidic protein staining $(\times 200)$. C: Calcific lesion with woven bone formation and fibrous area $(\mathrm{H} \& \mathrm{E}, \times 40)$. 
nosis of a neuroglialchoristoma was made based on a diffuse immunopositive reaction for glial fibrillary acidic protein (GFAP, mouse monoclonal antibody, Clone, DP46.10; diluted, 1:100, Signet; Dedham, MA, USA) (Fig. 3B). The diffusely calcified lesion showed woven bone formation and scant fibrous tissue consistent with the diagnosis of tympanosclerosis (Fig. 3C).

\section{Discussion}

The most common type of choristoma in the mastoid or middle ear is the salivary choristoma and the rarest is the neuroglialchoristoma. Neuroglialchoristomas are often classified according to their location and possible aetiopathogenesis as follows: intraparenchymalcentral nervous system lesions, dural and leptomeningeal lesions, intracranial extracerebral lesions, distal lesions considered to result from a previous pregnancy, midline nasal gliomas and non-midline lesions of the head and neck. Based on our literature review, neurogliomas of the middle ear and mastoid have previously been described in only 32 cases. Symptoms of choristoma of the middle ear include hearing loss, otorrhea, ear fullness, tinnitus and otalgia. Although the etiology and development process of neuroglial choristomas are unclear, the most accepted hypothesis is the inhalation theory. According to this theory, brain tissue herniation known as encephalocele may occur as a spontaneous lesion or as a result of bone and dural defects by trauma, infection, or neoplasm. When a pedicle directly connecting the neuroglial tissue with the subarachnoid or ventricular spaces may become detached and eventually get absorbed or become vestigial, an isolated ectopic mass of brain tissue develops. Based on this theory, neuroglial choristomas consist of a special type of encephalocele, wherein the connection with the brain disappears [4]. This hypothesis is supported by several predisposing factors likely to cause defects in the tegmen tympani, including a previous trauma or surgery or concomitant infection. However, our literature review revealed that defects in the tegmen based on pre-operative CT or intraoperative findings were reported in only $8 / 32$ cases [5-11]. Direct evidence of a connection with the brain parenchyma, such as an intraoperative CSF leak, was confirmed in only $2 / 32$ cases [7]. Concomitant chronic or recurrent otitis media was reported in 15/32 cases, including 3 with cholesteatoma [9]; a history of surgery or trauma was reported in 6/32 cases [7,9]. This implies that most cases of choristomas may not develop by inhalation of the brain tissue. Another hypothesis was that an error in early embryonic development occurs through the displacement of neuroectodermal cells capable of differentiation and proliferation. At a distant location, these cells then form a mass of ectopic brain tissue resembling a neural crest remnant [12]. Several such cases at a very young age with intact tympanic membranes and no history of otitis media advocate high possibilities of congenital development.

According to the inhalation theory on the development of neuroglial choristomas, the tegmen defect seems to be an important process. Spontaneous tegmen defects are less common in adults, and chronic otitis media with or without cholesteatoma is the most common cause of tegmen defects in adults. Other causes include temporal bone trauma, neoplasia, and radiotherapy [13]. Meanwhile, eustachian tube dysfunction and poor aeration of the middle ear cavity are the major predisposing factors of not only chronic otitis media [14] but also tympanosclerosis. Otitis media with or without effusion may lead to a destructive process within the connective tissue of the middle ear, which leads to the degeneration of collagen, subsequent dystrophic calcification, and tympanosclerosis [15]. Wielinga, et al. [3] demonstrated this using an experimental animal model of tympanosclerosis induced by Eustachian tube dysfunction, wherein the dysfunctioninduced prolonged otitis media with effusion, tympanic membrane fibril degeneration, an increase in fibroblast activity, abnormal fibrils formation and subsequent large hyalinized plaque formation. In our present case, negative pressure by eustachian tube dysfunction may have induced chronic otitis media, causing the tegmen defect followed by subsequent development of choristoma. Simultaneously, long-lasting negative pressure and chronic inflammation are assumed to induce massive tympanosclerosis. Based on this putative pathogenesis, neuroglial choristoma combined with tympanosclerosis of the middle ear could be a case that strongly supports the existing inhalation theory.

\section{Conflicts of interest}

The authors have no financial conflicts of interest.

\section{REFERENCES}

1) Batsakis JG. Pathology consultation. Nomenclature of developmental tumors. Ann Otol Rhinol Laryngol 1984;93:98-9.

2) Heffner DK. Brain in the middle ear or nasal cavity: heterotopia or encephalocele? Ann Diagn Pathol 2004;8:252-7.

3) Wielinga EW, Kuijpers W, Tonnaer EL, Jap PH. An experimental model for tympanosclerosis. A preliminary report. Acta Otolaryngol 1988;105:537-42.

4) Plontke SK, Preyer S, Pressler H, Mundinger PM, Plinkert PK. Glial lesion of the infratemporal fossa presenting as a soft tissue middle ear mass-rudimentary encephalocele or neural crest remnant? Int J Pediatr Otorhinolaryngol 2000;56:141-7.

5) Martinez-Peñuela A, Quer S, Beloqui R, Bulnes MD, Martinez-Peñuela JM. Glial choristoma of the middle ear: report of 2 cases. Otol Neurotol 2011;32:e26-7.

6) Dunham E, Armeni M. Glial choristoma of the temporal bone in a 
7-month-old infant. JAMA Otolaryngol Head Neck Surg 2013;139: 944-6.

7) Gyure KA, Thompson LD, Morrison AL. A clinicopathological study of 15 patients with neuroglial heterotopias and encephaloceles of the middle ear and mastoid region. Laryngoscope 2000;110:1731-5.

8) Lee JI, Kim KK, Park YK, Eah KY, Kim JR. Glial choristoma in the middle ear and mastoid bone: a case report. J Korean Med Sci 2004;19:155-8.

9) McGregor DH, Cherian R, Kepes JJ, Kepes M. Case reports: heterotopic brain tissue of middle ear associated with cholesteatoma. Am J Med Sci 1994;308:180-3.

10) Shemanski KA, Voth SE, Patitucci LB, Ma Y, Popnikolov N, Katsetos CD, et al. Glial choristoma of the middle ear. Ear Nose Throat J 2013;92:555-7.

11) Uğuz MZ, Arslanoğlu S, Terzi S, Etit D. Glial heterotopia of the middle ear. J Laryngol Otol 2007;121:e4.

12) Drapkin AJ. Rudimentary cephalocele or neural crest remnant? Neurosurgery 1990;26:667-73; discussion 674.

13) Lundy LB, Graham MD, Kartush JM, LaRouere MJ. Temporal bone encephalocele and cerebrospinal fluid leaks. Am J Otol 1996; 17:461-9.

14) Alper CM, Tabari R, Seroky JT, Doyle WJ. Magnetic resonance imaging of the development of otitis media with effusion caused by functional obstruction of the eustachian tube. Ann Otol Rhinol Laryngol 1997;106:422-31.

15) Chole RA. Chronic otitis media, mastoiditis, and petrositis. In: Flint PW, Haughey BH, Lund VJ, Niparko JK, Robbins KT, Thomas JR, et al. editors. Cummings otolaryngology--head \& Neck Surgery. 6th ed. Philadelphia: Saunders;2015. 